\title{
Effect of Plant Growth Regulators on Leaf Number, Leaf Area and Leaf Dry Matter in Grape
}

\author{
Zahoor Ahmad BHAT ${ }^{1}$, Rizwan RASHID², Javid Ahmad BHAT ${ }^{3}$ \\ ${ }^{1}$ Fruit Research Station Balapora, Skuast(K), Srinagar, India; zahoornano@gmail.com \\ ${ }^{2}$ Central Institute of Temperate Horticulture, Srinagar, India; rizwan60@gmail.com \\ ${ }^{3}$ Deptartament of Plant Pathology, PAU-Ludhaina India; javidrashid635@gmail.com
}

\begin{abstract}
Influence of phenylureas (CPPU) and brassinosteriod (BR) along with GA (gibberellic acid) were studied on seedless grape vegetative characteristics like leaf number, leaf area and leaf dry matter. Growth regulators were sprayed on the vines either once $(7$ days after fruit set or 15 days after fruit set) or twice (7+15 days after fruit set). CPPU 2 ppm+BR 0.4 ppm+GA 25 ppm produced maximum number of leaves (18.78) while as untreated vines produced least leaf number (16.22) per shoot. Maximum leaf area $(129.70 \mathrm{~cm} 2)$ and dry matter content (26.51\%) was obtained with higher CPPU (3 ppm) and BR (0.4 ppm) combination along with GA 25 ppm. Plant growth regulators whether naturally derived or synthetic are used to improve the productivity and quality of grapes. The relatively high value of grapes justifies more expensive inputs. A relatively small improvement in yield or fruit quality can justify the field application of a very costly product. Application of new generation growth regulators like brassinosteroids and phenylureas like CPPU have been reported to increase the leaf number as well as leaf area and dry matter thereby indirectly influencing the fruit yield and quality in grapes.
\end{abstract}

Keywords: brassinosteriod, growth regulator, leaf dry matter, leaf number, phenylureas

Abbreviations: BR-brasinosteroid, GA-gibberellic acid, CPPU-phenylurea, DAFS-days after fruit set

\section{Introduction}

In India diversity in climate, soil and manipulation of cultural practices helps to produce grapes throughout the year. Large quantities of fresh fruits in the farm of raisins are produced and exported to other countries. Besides soil and climate, the plant factors also play an important role in grape production. Among plant factors the photosynthetic area (leaf area) is very important. Purohit et al. (1979) reported that leaf area of $3064 \mathrm{~cm}^{2}$ was optimum for bunch and berry development is 'Anab-e-Shahi'. The best quality fruits were produced by maintaining 12-16 leaves per bunch in 'Anab-e-Shahi' grape (Patil and Nagpal, 1981). A specific number of leaves and leaf area are required for optimum growth and development of fruits in different crops. Hence the present investigation was carried out to evaluate the effect of new generation growth regulators on leaf parameters which ultimately influence the berry and bunch growth and development. Brassinosteroids (BRs) are a class of polyhydroxysteroids that have been recognized as a sixth class of plant hormones. These were first explored nearly forty years ago when Mitchell $e t$ al. (1972) reported promotion in stem elongation and cell division by the treatment of organic extracts of rapeseed (Brassica napus) pollen. Brassinolide was the first isolated brassinosteroid in 1979 when it was shown that pollen from Brassica napus could promote stem elongation and cell divisions, and the biologically active molecule was isolated. BRs have been shown to be involved in numerous plant processes like promotion of cell expansion and cell elongation synergism with auxin, play role in cell division and cell wall regeneration, promotion of vascular differentiation etc. CPPU are cytokinin like substances but have 100 times more potential than BA in promoting cell division. Both CPPU and BRs act synergistically with GA in promotion of many cellular processes including cell division and enlargement.

\section{Materials and methods}

The study was conducted on grape cv. 'Tas-A-Ganesh' in a commercial vineyard located in Hyderabad, when vines were seven year old. The vines were trained on overhead bower, planted at $3 \mathrm{~m} \times 1.5 \mathrm{~m}$ distance. There were three main treatments involving three different time intervals of growth regulator application and ten subplot treatments of different growth regulator combination with different concentration as follows:

- $\mathrm{T}_{1}$-Control

- $\mathrm{T}_{2}$-CPPU $2 \mathrm{ppm}$

- $\mathrm{T}_{3}$-CPPU 2 ppm+BR $0.2 \mathrm{ppm}+\mathrm{GA} 25 \mathrm{ppm}$

- $\mathrm{T}_{4}^{3}$ CPPU $2 \mathrm{ppm}+\mathrm{GA} 25 \mathrm{ppm}$ 
88

- $\mathrm{T}_{5}$-CPPU 2 ppm+BR $0.4 \mathrm{ppm}+\mathrm{GA} 25 \mathrm{ppm}$

- $\mathrm{T}_{6}^{5}$-CPPU 3 ppm

- $\mathrm{T}_{7}$-CPPU 3 ppm+BR $0.2 \mathrm{ppm}+\mathrm{GA} 25 \mathrm{ppm}$

- $\mathrm{T}_{8}-\mathrm{CPPU} 3 \mathrm{ppm}+\mathrm{GA} 25 \mathrm{ppm}$

- $\mathrm{T}_{9}$-CPPU 3 ppm+BR $0.4 \mathrm{ppm}+\mathrm{GA} 25 \mathrm{ppm}$

- $\mathrm{T}_{10}$-GA $25 \mathrm{ppm}$

Number of leaves per shoot were counted at veraison on five randomly selected shoots and average number per shoot was calculated. Leaf area meter was used to measure the leaf area of the leaves opposite to the cluster. Dry matter content was determined from the leaves used for area under each treatment. Fresh weight was calculated and were dried in oven at $70^{\circ} \mathrm{C}$ until a constant weight was recorded separately for each treatment at veraison. Dry matter content of leaves was then calculated as the percentage over fresh weight by following formula:

$$
\text { Dry matter content of leaves }=\frac{\text { Dry weight of leaves }}{\text { Fresh weight of leaves }} \times 100
$$

Data was subjected to ANOVA analysis using Latin Square Design (LSD)

\section{Results and discussion}

Number of leaves per shoot was significantly influenced by growth regulator treatments and their interactions. However, different times of growth regulator applications had no significant effect (Tab. 1). Combination of CPPU 2 ppm + BR 0.4 ppm + GA 25 ppm ( $\left.\mathrm{T}_{5}\right)$ recorded maximum number of leaves (18.78) per shoot which was at par with $\mathrm{T}_{3} \mathrm{~T}_{4}, \mathrm{~T}_{7}, \mathrm{~T}_{9}$ and $\mathrm{T}_{10}$. Whereas minimum leaf number per shoot was observed in control (16.22). Interaction effects also showed that $T_{5}$ at 7 DAFS had maxi- mum number of leaves (19.67) while $T_{1}$ and $T_{2}$ at $7+15$ DAFS recorded minimum leaf number.

There were significant differences among regulator treatments, time of their application as well as their interaction with regard to leaf area (Tab. 2). Among different time intervals, $7+15$ days after fruit set (DAFS) had significantly higher leaf area $\left(120.08 \mathrm{~cm}^{2}\right)$ compared to 7 DAFS and 15 DAFS $\left(126.7 \mathrm{~cm}^{2}\right)$ and $126.81 \mathrm{~cm}^{2}$ respectively. Growth regulator treatments showed that $\mathrm{T}_{9}$ had maximum leaf area $\left(129.710 \mathrm{~cm}^{2}\right)$ followed by $\mathrm{T}_{4}(128.66$ $\left.\mathrm{cm}^{2}\right)$. Whereas control $\left(\mathrm{T}_{1}\right)$ recorded minimum leaf area $\left(125.75 \mathrm{~cm}^{2}\right)$ and was at par with $\mathrm{T}_{2}, \mathrm{~T}_{3}, \mathrm{~T}_{5}, \mathrm{~T}_{6}, \mathrm{~T}_{7}$ and $\mathrm{T}_{10}$ Interaction effect revealed that $\mathrm{T}_{9}$ at $7+15$ DAFS had maximum leaf area $\left(130.65 \mathrm{~cm}^{2}\right)$

The perusal of data revealed that different times of application had no significant effect on leaf dry matter (Tab. 3) but among growth regulators higher phenylurea (CPPU) and brasinosteroid (BR) combination along with GA 25 ppm had maximum leaf dry matter percentage $(26.51 \%)$. Interaction was also significant and $7+15$ DAFS with $T_{9}$ recorded maximum value (27.67) whereas control $\left(\mathrm{T}_{1}\right)$ at 15 DAFS recorded lowest dry matter content (21.87).

Leaf number decreased with higher CPPU concentration (3 ppm) either alone or in combination with GA. However, lower CPPU (2 ppm) and higher BR (0.4 ppm) with GA 25 ppm exhibited maximum leaf number. Similar results were earlier reported by Kumar (1996). This may be due to inhibition of shoot growth by CPPU and diversion of metabolites for bunch development thereby reducing availability of metabolites for shoot elongation. Higher leaf area recorded with higher CPPU and BR concentrations in combination with GA may be due to increased concentration of photosynthates in the shoot (Iwahori et al., 1988; Kumar, 1964; Mitchell and George

Tab. 1. Effect of growth regulators at different days after fruit set (DAFS) on number of leaves per shoot in grape Cv.'Tas-AGanesh'

\begin{tabular}{|c|c|c|c|c|}
\hline Treatment & 7 DAFS & 15 DAFS & $7+15 \mathrm{DAFS}$ & Mean \\
\hline $\mathrm{T}_{1}$-Control & 16.67 & 15.67 & 16.33 & 16.22 \\
\hline $\mathrm{T}_{2}$-CPPU $2 \mathrm{ppm}$ & 17.67 & 17.33 & 16.33 & 17.11 \\
\hline $\mathrm{T}_{3}$-CPPU $2 \mathrm{ppm}+\mathrm{BR} 0.2 \mathrm{ppm}+\mathrm{GA} 25 \mathrm{ppm}$ & 18.67 & 18.33 & 18.00 & 18.33 \\
\hline $\mathrm{T}_{4}-\mathrm{CPPU} 2 \mathrm{ppm}+\mathrm{GA} 25 \mathrm{ppm}$ & 18.00 & 17.00 & 18.00 & 17.67 \\
\hline $\mathrm{T}_{5}-\mathrm{CPPU} 2 \mathrm{ppm}+\mathrm{BR} 0.4 \mathrm{ppm}+\mathrm{GA} 25 \mathrm{ppm}$ & 19.67 & 17.67 & 19.00 & 18.78 \\
\hline $\mathrm{T}_{6}-\mathrm{CPPU} 3 \mathrm{ppm}$ & 17.00 & 16.33 & 17.67 & 17.00 \\
\hline $\mathrm{T}_{7}-\mathrm{CPPU} 3 \mathrm{ppm}+\mathrm{BR} 0.2 \mathrm{ppm}+\mathrm{GA} 25 \mathrm{ppm}$ & 18.67 & 17.00 & 17.67 & 17.78 \\
\hline $\mathrm{T}_{8}-\mathrm{CPPU} 3 \mathrm{ppm}+\mathrm{GA} 25 \mathrm{ppm}$ & 17.00 & 17.33 & 17.33 & 17.22 \\
\hline $\mathrm{T}_{9}$-CPPU 3 ppm+BR $0.4 \mathrm{ppm}+\mathrm{GA} 25 \mathrm{ppm}$ & 17.67 & 17.67 & 18.33 & 17.89 \\
\hline $\mathrm{T}_{10}-\mathrm{GA} 25 \mathrm{ppm}$ & 17.67 & 17.33 & 17.67 & 17.56 \\
\hline Mean & 17.87 & 17.17 & 17.63 & --- \\
\hline Source of variation & \multicolumn{2}{|c|}{ S.Em $(+)$} & \multicolumn{2}{|c|}{$\mathrm{CD}(0.05)$} \\
\hline Main & \multicolumn{2}{|c|}{0.22} & \multicolumn{2}{|c|}{ NS } \\
\hline Sub & \multicolumn{2}{|c|}{0.771} & \multicolumn{2}{|c|}{.54} \\
\hline Sub at main & \multicolumn{2}{|c|}{1.33} & \multicolumn{2}{|c|}{2.67} \\
\hline & \multicolumn{2}{|c|}{0.79} & \multicolumn{2}{|c|}{1.60} \\
\hline
\end{tabular}


Tab. 2. Effect of growth regulators at different days after fruit set (DAFS) on leaf area( $\left(\mathrm{cm}^{2}\right)$ in grape Cv. 'Tas-A-Ganesh'

\begin{tabular}{|c|c|c|c|c|}
\hline Treatment & 7 DAFS & 15 DAFS & $7+15$ DAFS & Mean \\
\hline $\mathrm{T}_{1}$-Control & 125.42 & 125.42 & 126.42 & 125.75 \\
\hline $\mathrm{T}_{2}$-CPPU $2 \mathrm{ppm}$ & 125.01 & 126.85 & 127.53 & 126.47 \\
\hline $\mathrm{T}_{3}$-CPPU $2 \mathrm{ppm}+\mathrm{BR} 0.2 \mathrm{ppm}+\mathrm{GA} 25 \mathrm{ppm}$ & 128.50 & 125.13 & 127.28 & 127.07 \\
\hline $\mathrm{T}_{4}-\mathrm{CPPU} 2 \mathrm{ppm}+\mathrm{GA} 25 \mathrm{ppm}$ & 128.57 & 126.99 & 130.43 & 128.66 \\
\hline $\mathrm{T}_{5}-\mathrm{CPPU} 2 \mathrm{ppm}+\mathrm{BR} 0.4 \mathrm{ppm}+\mathrm{GA} 25 \mathrm{ppm}$ & 126.06 & 126.70 & 128.31 & 127.03 \\
\hline $\mathrm{T}_{6}-\mathrm{CPPU} 3 \mathrm{ppm}$ & 126.41 & 126.60 & 127.72 & 126.91 \\
\hline $\mathrm{T}_{7}$-CPPU 3 ppm+BR $0.2 \mathrm{ppm}+\mathrm{GA} 25 \mathrm{ppm}$ & 126.41 & 126.51 & 127.30 & 126.74 \\
\hline $\mathrm{T}_{8}-\mathrm{CPPU} 3 \mathrm{ppm}+\mathrm{GA} 25 \mathrm{ppm}$ & 126.49 & 127.99 & 128.77 & 127.75 \\
\hline $\mathrm{T}_{9}$-CPPU 3 ppm+BR $0.4 \mathrm{ppm}+\mathrm{GA} 25 \mathrm{ppm}$ & 129.54 & 128.92 & 130.65 & 129.70 \\
\hline $\mathrm{T}_{10}-\mathrm{GA} 25 \mathrm{ppm}$ & 125.58 & 126.38 & 126.39 & 126.11 \\
\hline Mean & 126.77 & 126.81 & 128.08 & $-\ldots-$ \\
\hline Source of variation & \multicolumn{2}{|c|}{ S.Em $(+)$} & \multicolumn{2}{|c|}{$\mathrm{CD}(0.05)$} \\
\hline Main & \multicolumn{2}{|c|}{0.36} & \multicolumn{2}{|c|}{0.89} \\
\hline Sub & \multicolumn{2}{|c|}{0.96} & \multicolumn{2}{|c|}{1.92} \\
\hline Sub at main & \multicolumn{2}{|c|}{1.66} & \multicolumn{2}{|c|}{3.33} \\
\hline Main at sub & \multicolumn{2}{|c|}{1.21} & \multicolumn{2}{|c|}{ NS } \\
\hline
\end{tabular}

Tab. 3. Effect of growth regulators at different days after fruit set (DAFS) on leaf dry matter content (\%) in grape Cv. 'Tas-AGanesh'

\begin{tabular}{|c|c|c|c|c|}
\hline Treatment & 7 DAFS & 15 DAFS & $7+15$ DAFS & Mean \\
\hline $\mathrm{T}_{1}$-Control & 22.09 & 21.84 & 22.31 & 22.08 \\
\hline $\mathrm{T}_{2}-\mathrm{CPPU} 2 \mathrm{ppm}$ & 23.92 & 24.30 & 24.05 & 24.09 \\
\hline $\mathrm{T}_{3}$-CPPU $2 \mathrm{ppm}+\mathrm{BR} 0.2 \mathrm{ppm}+\mathrm{GA} 25 \mathrm{ppm}$ & 24.83 & 25.25 & 25.78 & 25.29 \\
\hline $\mathrm{T}_{4}-\mathrm{CPPU} 2 \mathrm{ppm}+\mathrm{GA} 25 \mathrm{ppm}$ & 24.94 & 24.96 & 24.15 & 24.68 \\
\hline $\mathrm{T}_{5}$-CPPU $2 \mathrm{ppm}+\mathrm{BR} 0.4 \mathrm{ppm}+\mathrm{GA} 25 \mathrm{ppm}$ & 25.67 & 25.60 & 24.83 & 25.37 \\
\hline $\mathrm{T}_{6}-\mathrm{CPPU} 3 \mathrm{ppm}$ & 24.66 & 25.24 & 25.32 & 25.07 \\
\hline $\mathrm{T}_{7}-\mathrm{CPPU} 3 \mathrm{ppm}+\mathrm{BR} 0.2 \mathrm{ppm}+\mathrm{GA} 25 \mathrm{ppm}$ & 25.60 & 25.67 & 25.76 & 25.71 \\
\hline $\mathrm{T}_{8}-\mathrm{CPPU} 3 \mathrm{ppm}+\mathrm{GA} 25 \mathrm{ppm}$ & 24.56 & 25.26 & 25.44 & 25.09 \\
\hline $\mathrm{T}_{9}-\mathrm{CPPU} 3 \mathrm{ppm}+\mathrm{BR} 0.4 \mathrm{ppm}+\mathrm{GA} 25 \mathrm{ppm}$ & 25.79 & 26.04 & 27.67 & 26.51 \\
\hline $\mathrm{T}_{10}-\mathrm{GA} 25 \mathrm{ppm}$ & 24.42 & 24.38 & 24.76 & 24.52 \\
\hline Mean & 24.65 & 25.03 & 24.84 & $-\cdots$ \\
\hline Source of variation & \multicolumn{2}{|c|}{$\operatorname{S.Em}(+)$} & \multicolumn{2}{|c|}{$\mathrm{CD}(0.05)$} \\
\hline Main & \multicolumn{2}{|c|}{0.46} & \multicolumn{2}{|c|}{ NS } \\
\hline Sub & \multicolumn{2}{|c|}{0.95} & \multicolumn{2}{|c|}{1.90} \\
\hline Sub at main & \multicolumn{2}{|c|}{1.64} & \multicolumn{2}{|c|}{3.30} \\
\hline Main at sub & \multicolumn{2}{|c|}{1.47} & \multicolumn{2}{|c|}{ NS } \\
\hline
\end{tabular}

1972; Nunez et al., 1998; Zoffoli et al., 2009).Combination of CPPU and BR either at low or high concentrations along with GA 25 ppm resulted in increased leaf dry matter content. This increase in leaf dry matter may be attributed to increase in leaf area by CPPU and BR as is evident from positive relation between leaf area and leaf dry matter content. In addition to brassinosteroids, phenylureas like CPPU (N-(2-chloro-4-pyridyl)-N'-phenylurea) commonly called as forehlorofenuron have been synthesized (Zullo and Kohout, 2004). Both these compounds when used at submolar concentrations have been shown to stimulate various physiological and biochemical responses in many plants (Sasse, 2003).

\section{Conclusions}

Application of phenylureas (CPPU) and brassinosteriod (BR) along with GA affected vegetative characteristics like leaf number, leaf area and leaf dry matter in seedless grape cultivar Tas-A-Ganesh. When CPPU 2 ppm+BR $0.4 \mathrm{ppm}+\mathrm{GA} 25 \mathrm{ppm}$ was sprayed twice i.e once at 7 DAFS and again at 15 DAFS maximum number of leaves (18.78) per shoot were obtained while maximum leaf area $\left(129.70 \mathrm{~cm}^{2}\right)$ and dry matter content $(26.51 \%)$ was obtained with higher CPPU (3 ppm) and BR ( 0.4 ppm) concentration in combination with GA $25 \mathrm{ppm}$. This clearly indicates that the new generation growth regulators have 
90

a potential to be used in future for improvement of grape quality, especially in seedless varieties so as to tape the international markets.

\section{References}

Iwahori S, Tominga S, Yamasaki T (1998). Stimulation of fruit growth of Kiwi fruit by N-(2-Chloro-4-pyridyl)-Nphenylurea derivative cytokinins. HortScience 35:109-115.

Kumar KA (1996). Studies on improving bunch characteristic in seedless cultivars of grapes. MSc. Thesis, UAS, Bangalore.

Kumar MN (1964). Influence of leaf area and age of wood on yield and quality of some varieties of grapes. M.Sc. Thesis submitted to TNAU-Coimbatore.

Mitchell JW, George CE (1972). Enhancement of overall plant growth,a new response to brassins. Nature 239:253-254.

Nunez M, Musa JL, Alfonso JL, Coll F (1998). The influence of two new Cuban Bioregulators on plant yield of onion(Allium сера) cv. 'Red Creole.' Cultivas Trop 19:21-24.
Patil BA, Nagpal RL (1981). Effect of different number of leaves per bunch on growth, yield and quality of 'Anab-e-Shahi' grape. Drksharatha 1:64.

Purohit AG, Shikhamany SD, Prasanakumar B (1979).Effect of number of leaves per bunch on growth and quality of 'Anabe-Shahi'. Ind J Hort 36:36-41.

Sasse JM (2003). Physiological actions of brassinosteroids; an update. J Plant Growth Regul 22:276-288.

Zoffoli JP, Latorre BA, Naranjo P (2009). Preharvest application of growth regulators and their effect on postharvest quality of table grapes during cold storage. Post Harvest Biology and Technology 51:183-192.

Zullo MAT, Kohout L (2004). Semisystematic nomenclature of brassinosteroids. J Plant Growth Regul 42:15-28. 